\title{
An Efficient Scheme for Onboard Reduction of Moored $\chi$ pod Data
}

\author{
JOHANNES BECHERER AND JAMES N. MOUM \\ College of Earth, Ocean, and Atmospheric Sciences, Oregon State University, Corvallis, Oregon
}

(Manuscript received 30 June 2017, in final form 23 August 2017)

\begin{abstract}
A scheme for significantly reducing data sampled on turbulence devices ( $\chi$ pods) deployed on remote oceanographic moorings is proposed. Each $\chi$ pod is equipped with a pitot-static tube, two fast-response thermistors, a three-axis linear accelerometer, and a compass. In preprocessing, voltage means, variances, and amplitude of the $f^{1 / 3}$ subrange (inertial-convective subrange of the turbulence) of the voltage spectrum representing the temperature gradient are computed. Postprocessing converts voltages to engineering units, in particular mean flow speed (and velocity), temperature, temperature gradient, and the rate of destruction of the temperature variance $\chi$ from which other turbulence quantities, such as heat flux, are derived. On 10-min averages, this scheme reduces the data by a factor of roughly 24000 with a small (5\%) low bias compared to complete estimates using inertial-convective subrange scaling of calibrated temperature gradient spectra.
\end{abstract}

\section{Introduction}

Recent advances in moored mixing measurements using $\chi$ pods have led to long time series records from a range of locations. From a fast temperature measurement that incompletely resolves the temperature variance associated with turbulence-scale fluctuations (Moum and Nash 2009; Zhang and Moum 2010; Perlin and Moum 2012), we have obtained continuous records up to 18 months long. These have yielded some insight into the details of the shear instabilities in the marginally unstable stratified shear flow above the Equatorial Undercurrent (Moum et al. 2011; Smyth et al. 2011; Smyth and Moum 2013), a resolution of mixing's contribution to the annual cycle of SST in the equatorial cold tongue despite semiannual peaks in surface heating (Moum et al. 2013), an initial look at monsoon mixing cycles (Warner et al. 2016), and a proposed new ocean-atmosphere feedback mechanism to the Madden-Julian oscillation in the equatorial Indian Ocean (Moum et al. 2016).

In this effort, we have attempted to maintain continuous multiyear records at several locations, particularly in the equatorial cold tongue of the Pacific. One limitation has been mooring losses. NOAA's National Data Buoy Center maintains these moorings and returns reduced data back via inductive modem (up the

Corresponding author: Johannes Becherer, jbecherer@coas. oregonstate.edu cable) and by satellite to our desktops in almost real time via its website (http://tao.ndbc.noaa.gov/).

$\chi$ pods sample a range of signals at roughly 1200 bytes per second. While learning how to make sense of the $\chi$ pod data, it has been essential to use all of these data to ensure optimal computations of turbulence quantities. However, no data are returned from lost moorings. In an attempt to remedy this, we investigate schemes for efficient reduction of data from $\chi$ pods that can be later applied in firmware for near-real-time data transmission to our laboratory, where postcalibration and postprocessing can be done to derive turbulence quantities. We demonstrate this by postprocessing an existing dataset in a way that might be implemented in microprocessor firmware. In the text, we specifically refer to preprocessing as the analysis that would be done on board $\chi$ pods and to postprocessing as the analysis performed on voltage means and variances computed in preprocessing.

In this paper we first summarize how we quantify turbulence measurements from $\chi$ pods (section 2) and then briefly describe the particular measurements used for the evaluation to follow (section 3). We compare estimates of turbulence quantities in high- and lowwavenumber regimes of the turbulence spectrum in section 4. Our schemes require reducing several quantities to voltage averages and/or variances. Reduction of the velocity signal derived from pitot-static tubes is developed in section 5, where it is also compared to a nearby reference velocity. In section 6 , we develop the 
scheme for calibrating reduced temperature and its gradient. Two schemes to compress and then to calibrate spectral information with units $\mathrm{V}^{2} \mathrm{~Hz}^{-1}$ are introduced in section 7 and are evaluated in section 8 . A brief discussion of the applicability of our method in an anisotropic regime follows in section 9, and conclusions are in section 10.

\section{Turbulence measurements with $\chi$ pods}

In this section we briefly describe the rationale and methods to derive quantitative turbulence information from moored $\chi$ pod measurements (for details see Moum and Nash 2009). $\chi$ pods are equipped with two fastresponse FP07 temperature sensors, a pressure sensor, a pitot-static tube to detect the mean flow speed past the sensor, linear accelerometers to independently measure cable-induced motions of the $\chi$ pod, and a compass to convert speed to velocity (Table 1 ). To translate the high-frequency temperature information into turbulence data, we start with the transport equation for temperature variance (Pope 2000),

$$
\frac{d\left\langle\left(T^{\prime}\right)^{2}\right\rangle}{d t}+\mathscr{T}_{T}=\mathscr{P}_{T}-\chi,
$$

where $T^{\prime}$ denotes turbulent temperature fluctuations in a Reynolds-averaged framework, $\langle\cdot\rangle$ represents an ensemble average that we estimate as a time average, and $\mathscr{T}_{T}$ combines all possible transport terms. On the right-hand side of (1) are the source and sink terms for temperature variance. If isotherms are horizontal, then the production of temperature variance can be written as

$$
\mathscr{P}_{T}=-2\left\langle w^{\prime} T^{\prime}\right\rangle T_{z}
$$

and parameterized by a turbulent diffusivity $K_{T}$ as

$$
\mathscr{P}_{T}=2 K_{T} T_{z}^{2},
$$

where subscript $z$ represents differentiation in the vertical and $w$ is the vertical component of velocity. The sink term in (1) is the temperature variance decay,

$$
\chi=2 D_{T}\left\langle\left(\frac{d T^{\prime}}{d x_{i}} \frac{d T^{\prime}}{d x_{i}}\right)\right\rangle,
$$

which is the irreversible diffusion of temperature variance by molecular mixing at small scales. In (4) $D_{T}$ is the molecular diffusivity of heat and $i$ represents three spatial dimensions, where we use the Einstein convention to sum over repeated indices. Since $\chi$ is strictly positive, it is always a sink term in (1).
TABLE 1. Quantities measured by $\chi$ pods.

\begin{tabular}{lcc}
\hline \hline \multicolumn{1}{c}{$\chi$ pod measures } & Variable & $\begin{array}{c}\text { Sampling rate } \\
\text { (samples per second) }\end{array}$ \\
\hline Time & $t$ & 50 \\
Temperature & $T_{1}, T_{2}$ & $2 \times 50$ \\
Temperature derivative & $T_{1}^{\prime}, T_{2}^{\prime}$ & $2 \times 100$ \\
Pressure & $P$ & 50 \\
Acceleration & $A_{x}, A_{y}, A_{z}$ & $3 \times 50$ \\
Pitot speed & $u_{\text {pitot }}$ & 50 \\
Compass & $\mathrm{cmp}$ & 4 \\
Sum & $\Sigma$ & 604 \\
\hline
\end{tabular}

The term $\chi$ is directly estimated from $\chi$ pod measurements. We represent the ensemble average of the temperature gradient fluctuations in (4) as an integral in wavenumber space of the power spectral density of the horizontal temperature gradient $\Phi_{T_{x}}$,

$$
\chi=6 D_{T} \int_{0}^{\infty} \Phi_{T_{x}}(k) d k,
$$

where we assume the isotropy of small-scale temperature gradients. The horizontal temperature gradient is estimated from the measured temporal gradient via Taylor's frozen flow hypothesis (Taylor 1935),

$$
T_{x}=\left\langle u_{s}\right\rangle^{-1} T_{t},
$$

and

$$
k=2 \pi\left\langle u_{s}\right\rangle^{-1} f,
$$

where $\left\langle u_{s}\right\rangle$ denotes the average flow speed past the sensor.

It has to be noted that we do not calculate the entire integral in (5) with just the $\chi$ pod data itself for two reasons. First, the spectrum is not fully resolved when either $u_{s}$ or $\varepsilon$ is sufficiently large, both of which shift the spectrum to higher frequencies (or equivalent wavenumbers) than are resolved with fast thermistors. Second, some portion of the spectrum is contaminated by surface wave-induced motion of the mooring cable. Rather than integrating the measured spectrum, $\Phi_{T_{x}}(k)$ is compared to the theoretical spectrum proposed by Batchelor (1959) and scaled over a finite bandwidth of $f$ or $k$.

For our purposes, the relevant parts of the spectrum can be expressed theoretically as

$$
\Phi_{T_{x}}(k)=\chi[\overbrace{C_{\tau} \varepsilon^{-1 / 3} k^{1 / 3}}^{\text {IC-range }}+\overbrace{q \nu^{1 / 2} \varepsilon^{-1 / 2} k}^{\text {VC-range }}],
$$

with the molecular viscosity $\nu$ as well as with the constants $q=2.3$ (Klymak and Moum 2007) and $C_{\tau} \approx 0.4$ 


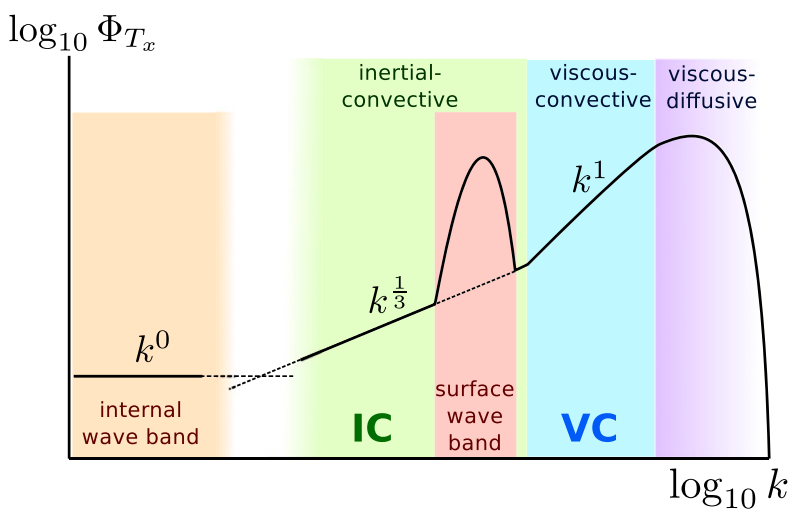

FIG. 1. Schematic of the temperature gradient spectrum.

(Sreenivasan 1996). A schematic of this spectrum is illustrated in Fig. 1.

Batchelor (1959) identifies the following three subranges for the turbulent tracer spectrum:

1) the inertial-convective subrange (IC), where we find a characteristic cascade toward smaller scales independent of molecular viscosity or diffusivity,

2) the viscous-convective subrange (VC), where viscosity becomes important and TKE is dissipated, and

3) the viscous-diffusive subrange, where molecular diffusivity actively diffuses the small-scale temperature gradients, causing irreversible mixing. ${ }^{1}$

At wavenumbers smaller than $k_{N}=N / u_{s}$, corresponding to the Brunt-Väisälä frequency $N$, internal waves dominate the spectrum. Surface waves contribute in a relatively narrow band, corresponding to periods between 2 and $20 \mathrm{~s}$. The viscous-diffusive regime is rarely resolved.

To fit the measured $\Phi_{T_{x}}$ to (8), we need to choose a wavenumber band. Moum and Nash (2009) scaled the VC range and Zhang and Moum (2010) showed the feasibility of IC scaling. Both scalings are implemented in the current versions of analysis software.

With the assumptions that turbulence is stationary and homogeneous, and that we can neglect transport terms, $\mathscr{P}_{T}=\chi$, we follow Osborn and Cox (1972), in which

$$
K_{T}=\frac{1}{2} \chi T_{z}^{-2}
$$

Osborn (1980) proposed a relation between the buoyancy flux $B$ and the dissipation rate of turbulence kinetic energy $\varepsilon$,

$$
B=K_{\rho} N^{2}=\Gamma \varepsilon,
$$

\footnotetext{
${ }^{1}$ The viscous-diffusive subrange is usually modeled with an exponential decrease in $k$ (Kraichnan 1968), but we do not include it in (8) because it is not used in this manuscript.
}

where the mixing efficiency is assumed to be constant, $\Gamma=0.2$. In the case where the turbulent diffusivities of density and temperature are equal, $K_{\rho}=K_{T}$, we can combine (9) and (10) to obtain an expression for the dissipation rate in terms of $\chi$,

$$
\varepsilon=\frac{N^{2}}{2 \Gamma T_{z}^{2}} \chi
$$

In other publications, this has been referred to as $\varepsilon_{\chi}$ to signify the origin of the estimate and to distinguish it from the more direct estimate obtained from airfoil probes. Here, we drop the subscript ${ }_{\chi}$, as we refer only to estimates based on $\chi$.

In summary, we require these four basic ingredients to follow the recipe described above:

1) high-frequency temperature measurements to get $T_{t}$,

2) the mean speed past the temperature sensor $u_{s}$ to convert frequency to wavenumber and temporal to spatial gradients [(6)],

$3)$ the vertical temperature gradient $T_{z}$ to calculate (9), and

4) the vertical stratification $N^{2}$ to estimate $\varepsilon$ [(11)].

\section{Measurements}

The data used for testing our data reduction schemes were obtained from a 1-yr-long $\chi$ pod deployment $30 \mathrm{~m}$ below the surface on a mooring at $0^{\circ} \mathrm{N}, 23^{\circ} \mathrm{W}$. This mooring is part of the equatorial Atlantic PIRATA array (Bourles et al. 2008) and was deployed on 14 April 2014 by R/V Le Suroit and recovered on 24 March 2015 by Bernard Bourles and colleagues at the Laboratoire d'Etudes en Géophysique et Océanographie Spatiales of the Institut de Recherche pour le Développement (IRD), France. The mooring was also equipped with temperature loggers and other instrumentation not discussed in this paper.

In addition, we use ADCP data from a collocated subsurface mooring maintained by Peter Brandt and colleagues at the GEOMAR Helmholtz Centre for Ocean Research Kiel, Germany. The ADCP (RD Instruments Workhorse, $150 \mathrm{kHz}$ ) was mounted at approximately 220-m depth, from 4 May 2014 to 23 September 2015.

Because of the technical nature of this manuscript we concentrate on one month of data, even though the $\chi$ pod yielded an entire year of high-quality data. We chose the month of June 2014 because it had the largest range in oceanographic conditions. During June 2014, the stratification varied by two orders of magnitude over a period of several days. The mean flow speed changed significantly from 0 to $>0.5 \mathrm{~m} \mathrm{~s}^{-1}$. As well, surface forcing and hence the wave field changed substantially. 
This diversity of conditions is important as a test of robustness of the proposed data reduction method to changing environmental conditions.

\section{VC versus IC estimates}

One way to estimate $\chi$ is to scale $\Phi_{T_{x}}(k)$ in the VC range [Eq. (8); Fig. 1], using 1-s-long temperature spectra, which means that estimates are based on a wavenumber range higher than that of the surface wave band (for details see Moum and Nash 2009). Since this is a fraction of a wave period, the variation in $u_{s}$ caused by wave-induced motion is minimized. Corresponding turbulence quantities estimated in the course of this manuscript will be referred to as VC estimates. The advantages of the VC fit are the avoidance of the surface wave band and some assurance that over that short period the turbulence is stationary. It also yields high temporal resolution. $\mathrm{VC}$ estimates of $\chi$ and $\varepsilon$ are typically further averaged to $1-10$-min intervals. The major disadvantage of this procedure is that it involves far too much data to send back via satellite.

Alternatively, we can scale $\Phi_{T_{x}}(k)$ at lower wavenumbers. Zhang and Moum (2010) demonstrated the use of the IC range to fit the temperature gradient spectrum to obtain values of $\chi$ that agree well with the VC estimates. The authors used a spectral coherence method to filter out the contributions of surface gravity waves.

Here, we use a slightly easier and more straightforward approach, avoiding the surface wave band, by scaling the IC part of the spectrum at frequencies (wavenumbers) less than the surface gravity wave band (Fig. 1). To be precise, we use spectra based on 10-minlong temperature data (at $100 \mathrm{~Hz}$ ) and we fit them over a wavenumber range that corresponds to $1 / 200-1 / 20 \mathrm{~Hz}$ in frequency space. Hence, we use only periods larger than $20 \mathrm{~s}$, thereby avoiding surface waves.

A particular advantage of these low wavenumber IC estimates is the requirement of significantly less data than the VC estimates. However, the wavenumber range extends to lower values of $k$, to a range where we might not expect isotropic turbulence, thus violating one of the assumptions described in section 2 . This is briefly reviewed in section 9 .

Figure 2 shows a comparison of $\chi$ estimated by scaling VC and IC subranges. We find that the methods agree well most of the time, with a correlation of more than $85 \%$ for the observation period (Fig. 2c). IC estimates greater than $\mathrm{VC}$ estimates correspond to small vertical temperature gradients $\left(T_{z}<10^{-2} \mathrm{~K} \mathrm{~m}^{-1}\right)$ (cf. Figs. 2a,b). In the 2D histogram, the systematic disagreement is seen in the tail of low $\chi^{\mathrm{VC}}$ values $\left(<10^{-7} \mathrm{~K}^{2} \mathrm{~s}^{-1}\right)$ associated with nearly constant $\chi^{\mathrm{IC}}$ values (Fig. 2c).
For both $\chi^{\mathrm{IC}}$ and $\chi^{\mathrm{VC}}$ we used the stratification estimates $T_{z}$ and $N^{2}$ calculated from the $\chi$ pod T signal (for details see Perlin and Moum 2012). Therefore, the difference between $\chi^{\mathrm{IC}}$ and $\chi^{\mathrm{VC}}$ apparent in Fig. 2 is primarily due to the choice of frequency band for (5).

\section{Speed}

As noted in section 2, the second major ingredient for estimating $\chi$ from $\chi$ pod is the flow speed past the sensor. The flow speed past the sensor $u_{s}$ is the vector sum of the ocean current speed $u_{b}$ plus the speed of the sensor induced by cable motion excited by the surface buoy. The latter is the motion of the $\chi$ pod relative to the fixed reference frame, defined by internal acceleration sensors (Perlin and Moum 2012). The speed $u_{s}$ is defined as

$$
u_{s}^{2}=\left(u_{b}-u_{A x}\right)^{2}+u_{A y}^{2}+u_{A z}^{2},
$$

where $u_{A x}, u_{A y}$, and $u_{A z}$ are $\chi$ pod body speeds in three orthogonal directions determined by integrating accelerations in those directions. This must be done for any interval estimate of $\chi$ via VC or IC scaling. Our objective here is to minimize the data required to obtain robust estimates of $\chi$. In this section we first show how we define an averaged estimate of $u_{b}$ from pitot-static tube measurements on $\chi$ pods and then compare it to $u_{b}$ measured at a nearby moored ADCP. We then define a corrected rms estimate of $u_{s}$ and compare it to that determined from averaged instantaneous measurements.

\section{a. Estimating $u_{b}$ from pitot-static tube}

Here we describe the computation of current speed from $\chi$ pod's pitot-static tube, thus making the procedure of computing $\chi$ independent of external measurement (e.g., ADCPs). Our procedure aims to maximally reduce the amount of data transferred from the $\chi$ pod without losing the ability to reconstruct the vital mean velocity information. The procedure includes pretransmission onboard processing and posttransmission calibration.

The onboard procedure requires the following substeps:

1) The pitot-static tube raw voltages $V_{s}(t)(50 \mathrm{~Hz})$ are averaged over the desired time interval $(10 \mathrm{~min}$ in this paper).

2) Small outliers are removed (all values less than two standard deviations below the mean).

3) The corrected signal is reaveraged and stored for transmission.

To illustrate the onboard averaging procedure, Fig. 3a shows a 10-min-long time series of fully resolved $V_{s}$ with a 1-min close up in Fig. 3b. Variability in $V_{s}$ is dominated by the period of surface waves about the 

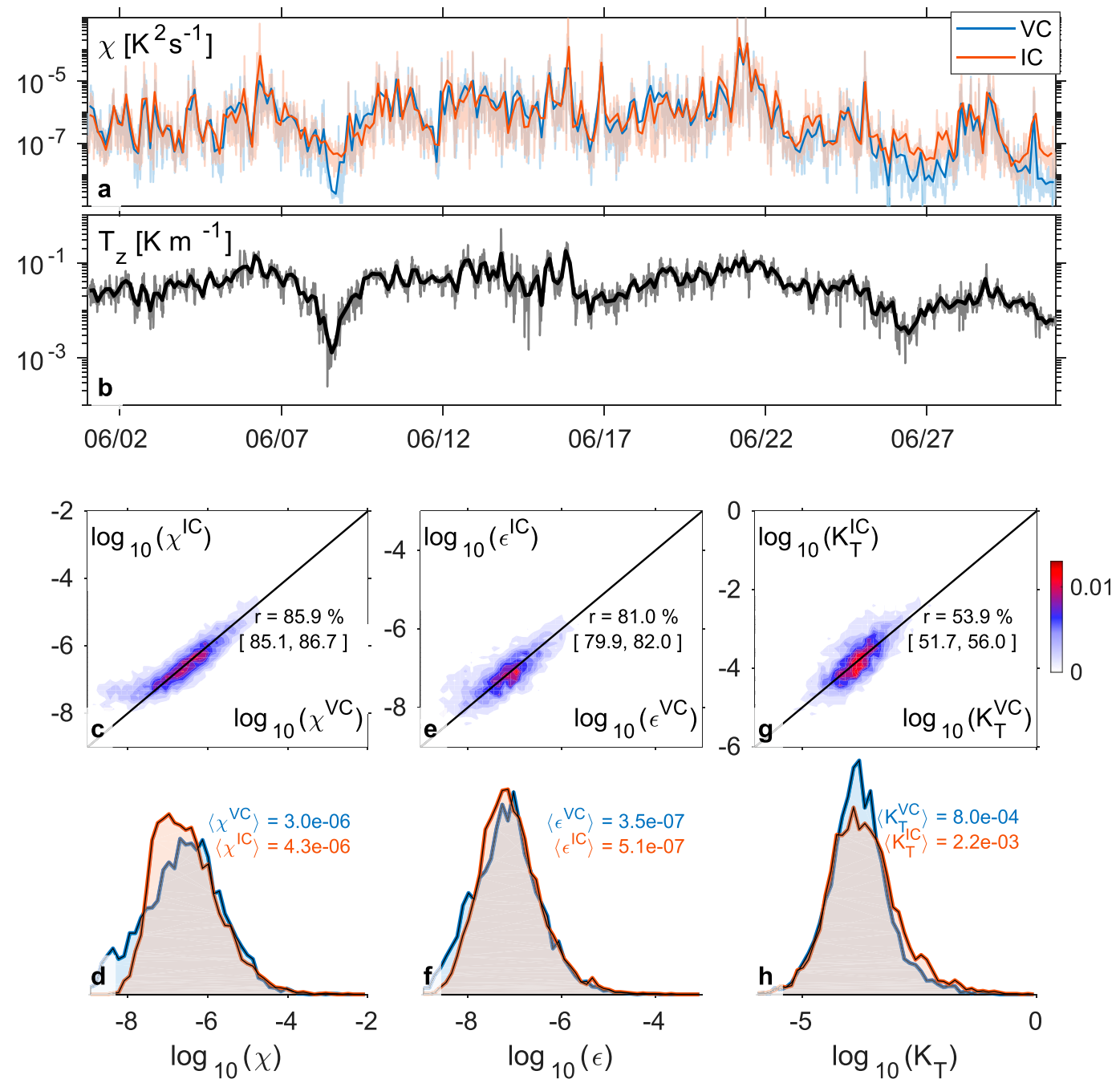

FIG. 2. Comparison between IC and VC estimates of $\chi$. (a) Time series of $\chi$ obtained from fits in the IC (red) and VC (blue) subranges. Shown are 3-h averages (solid lines) and 10-min estimates (shading). (b) Vertical temperature gradient (thick line: 3-h mean; shading: $10 \mathrm{~min}$ ). (c),(e),(g) 2D histograms of $\chi$, $\varepsilon$, and $K_{T}$, respectively. (d),(f),(h) 1D histograms corresponding to the panels above.

mean value. Occasional very low values are potentially caused by local flow reversals, where the pressure at the static port exceeds that at the dynamic port. To define an in situ value of the no-flow voltage $\left(C_{s 0}\right.$, defined by Moum 2015), these outliers are removed.

This procedure reduces $V_{s}$ to a 10 -min time average value $\bar{V}_{s}$. Further analysis to compute speed is done in postprocessing, in which we employ the following calibration procedure:

1) determine the no-flow voltage offset $C_{s 0}$ :

(a) correct $\bar{V}_{s}$ for temperature and pressure sensitivities variations $->\bar{V}_{s c}$ following the procedure of Moum (2015)

(b) find the smallest $5 \%$ of $\bar{V}_{s c}$ over the entire record (c) define $C_{s 0}$ as the median of these smallest $5 \%$

2) compute the speed $u_{b}^{p}=2 \rho^{-1}\left[C_{s}\left(\bar{V}_{s c}-C_{s 0}\right)\right]^{\frac{1}{2}}$, where $\rho$ is density and $C_{s}$ is the sensitivity of the pitot-static tube to variations in dynamic pressure

3) vectorize the speed into geographical coordinates utilizing the average compass direction measured by the $\chi$ pod.

The steps in the speed calibration procedure are illustrated in Fig. 4, where the 10-min-averaged values $\overline{V_{s c}}$ (light blue) are shown and used to determine $C_{s 0}$ (dashed brown line), resulting in the final voltage used to compute speed, $\overline{V_{s c}}-C_{s 0}$ (red). The pitot estimate of the current speed $u_{b}^{p}$ (orange, Fig. 4b) compares well to $u_{b}^{\text {adcp }}$ (blue, a reference speed from 


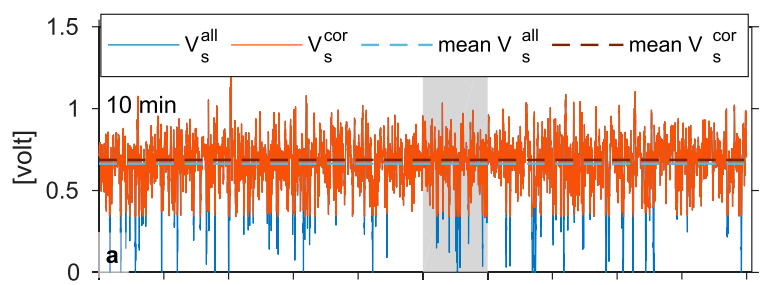

00:00 00:01 00:02 00:03 00:04 00:05 00:06 00:07 00:08 00:09 00:10

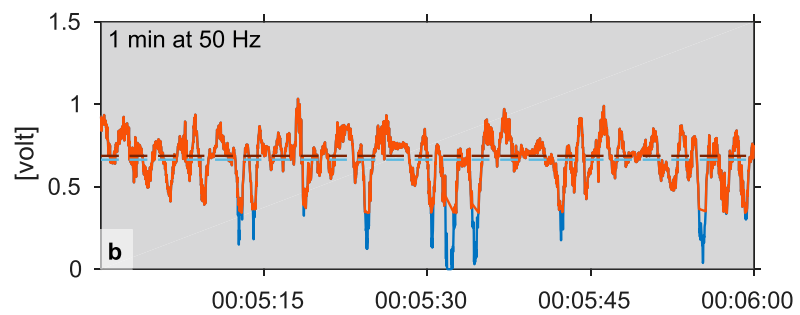

FIG. 3. (a) A 10-min time series of $V_{s}(50 \mathrm{~Hz})$ and (b) close up of a 1-min interval [gray shading in (a)]. Raw signal (blue line) with the corresponding 10-min average (dashed line). Remaining part of the signal after negative outliers been removed (red line), and the reaveraged value (red dashed line).

a nearby ADCP at the $\chi$ pod depth). With velocities inferred from accelerometers added, as described in section $5 \mathrm{c}$, the flow speed past the sensor is derived, $u_{s}$ (green).

\section{b. Comparison to ADCP data}

To confidently use our estimate of $u_{b}^{p}$, we first need to show that they compare well with available reference speed data. Moum (2015) showed that the pitot-static tube is able to reproduce 15-min-averaged velocities of ADCPs on subsurface moorings at 65- and 95-m depth. Here the situation is complicated by the shallower deployment depth $(30 \mathrm{~m})$ and the pumping induced by surface waves acting on the surface expression of the mooring. A much stronger wave contamination of the pitot-static signal ensues as a result of both stronger orbital velocities closer to the surface and the greater vertical movement of the instrument.

The comparison of both speeds and velocities (Fig. 5) measured from the pitot-static tube and ADCP suggests good agreement between the two instruments both in speed and direction. In terms of speed, the correlation is more than $75 \%$ with a small (5\%) high bias. Of all $u_{b}^{p}$ values, $95 \%$ agree within a factor of 2.5 with their corresponding $u_{b}^{\text {adcp }}$ value. Also, the direction information inferred from the $\chi$ pod compass agrees well with the flow direction of the ADCP. Given the fact that the ADCP is mounted on a different mooring several $100 \mathrm{~m}$ away and that the ADCP measures large spatial averages $[O(100) \mathrm{m}$ in the horizontal, $8 \mathrm{~m}$ in the vertical] as opposed to the point measurements of the pitot-static tube, the estimates agree reassuringly well. The fact that

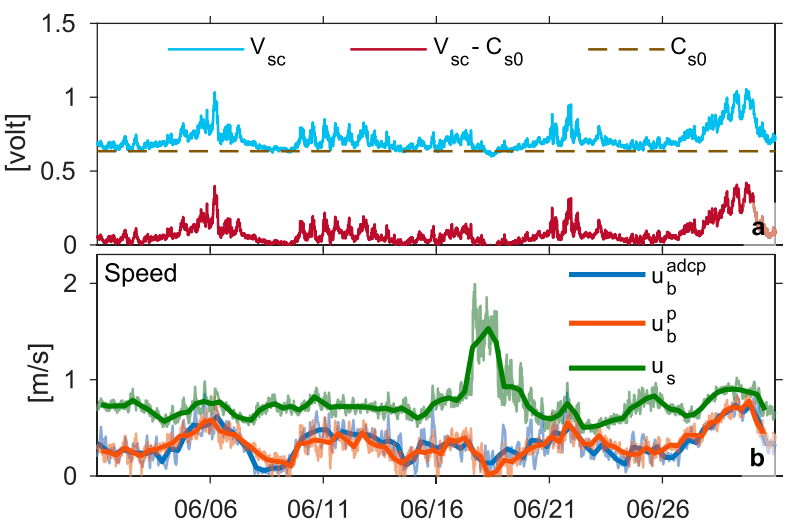

FIG. 4. (a) Shown are $\bar{V}_{\text {sc }}$, (blue), with $C_{s 0}$ subtracted (red), $C_{s 0}$ (brown). (b) Speed comparison $u_{b}^{p}$ (red), $u_{b}^{\text {adcp }}$ (blue), and the final $u_{s}$ obtained by combining the $u_{b}$ and cable speeds inferred from accelerations (green).

the ADCP is mounted on a subsurface mooring that is subject to blow over by currents could complicate a direct comparison of ADCP and pitot tube speeds. During the month of June 2014 the ADCP moved periodically (at the semidiurnal period) up and down with maximum displacement $<4 \mathrm{~m}$ in the vertical, or half an ADCP bin size. The tilt was less than $1^{\circ}$ throughout, and it was accounted for in the processing, such that we believe the movement of the ADCP mooring did not contribute significantly in our comparison.

\section{c. Estimating $u_{s}$}

To derive a corrected rms estimate of $u_{s}$, we first decompose it into a mean and deviation from that mean,

$$
u_{s}=\left\langle u_{s}\right\rangle+u_{s}^{\prime},
$$

where $\langle\cdot\rangle$ denotes the average and $(\cdot)^{\prime}$ denotes the deviation. The squared average speed past the sensor is

$$
\left\langle u_{s}\right\rangle^{2}=\left\langle u_{s}^{2}\right\rangle-\left\langle u_{s}^{\prime 2}\right\rangle
$$

where $2\left\langle\left\langle u_{s}\right\rangle u_{s}^{\prime}\right\rangle=0$, since $\left\langle u_{s}^{\prime}\right\rangle=0$. We now derive an exact equation for the first term on the right-hand side of (14) by averaging (12),

$$
\begin{aligned}
\left\langle u_{s}^{2}\right\rangle & =\left\langle\left(u_{b}-u_{A x}\right)^{2}\right\rangle+\left\langle u_{A y}^{2}\right\rangle+\left\langle u_{A z}^{2}\right\rangle \\
& =u_{b}^{2}-2 u_{b}\left\langle u_{A x}\right\rangle+\left\langle u_{A x}^{2}\right\rangle+\left\langle u_{A y}^{2}\right\rangle+\left\langle u_{A z}^{2}\right\rangle \\
& =u_{b}^{2}+\left\langle u_{A x}^{2}\right\rangle+\left\langle u_{A y}^{2}\right\rangle+\left\langle u_{A z}^{2}\right\rangle
\end{aligned}
$$

where $\left\langle u_{b}\right\rangle=u_{b}$ and the cable motion does not yield a residual velocity, that is, $\left\langle u_{A x}\right\rangle=0$. 

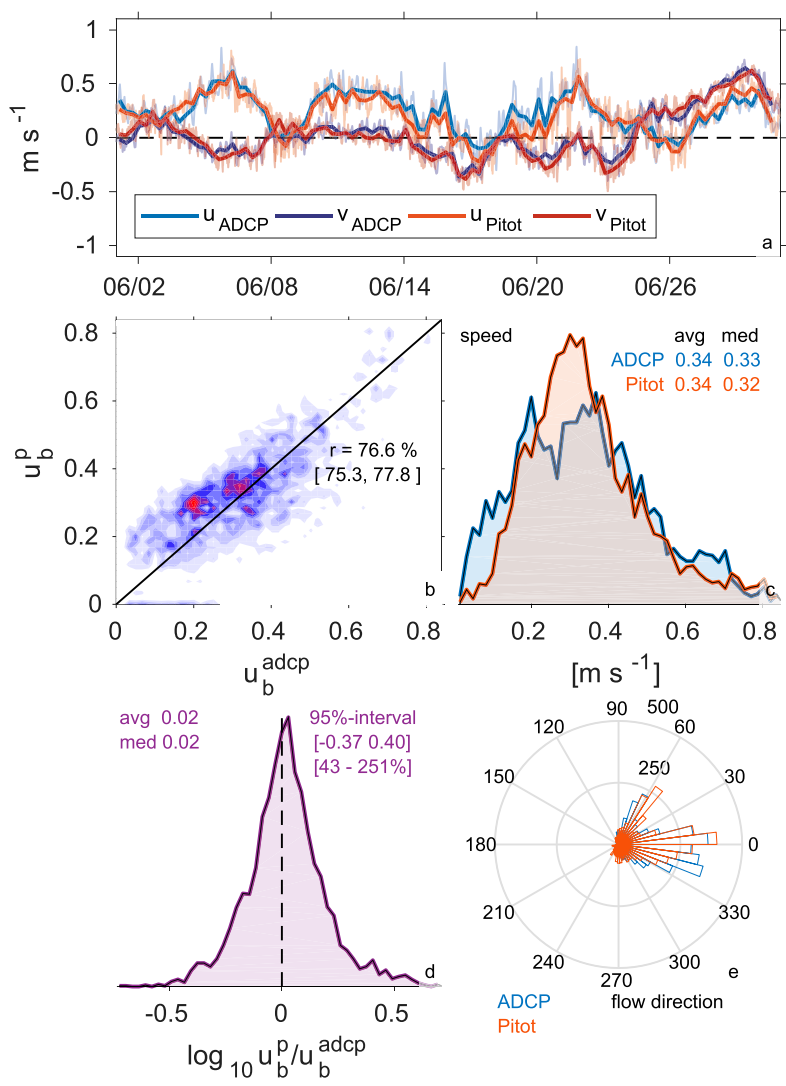

FIG. 5. Velocity comparison between ADCP (blue) and pitot tube (red). (a) Time series of the eastward $(u)$ - and northward $(v)$-directed velocity component (thick line: 12 -h average; thin lines: 1-h averages) (b) 2D histogram and (c) 1D histogram of speed. (d) Ratio between the different speed estimates. (e) Histogram of the flow direction.

The aim is to calculate $\left\langle u_{s}\right\rangle$ by solving (14). Since (15) is exact, the problem is to find an expression for $\left\langle u_{s}^{\prime 2}\right\rangle$. Term $u_{s}^{\prime}$ is completely due to wave-induced mooring cable motions. While it can be computed on time scales shorter than a wave period, it cannot be determined explicitly in terms of $\left\langle u_{A x}^{2}\right\rangle,\left\langle u_{A y}^{2}\right\rangle$, or $\left\langle u_{A z}^{2}\right\rangle$. We note that the coordinate cable speeds have both signs, while the variances are always $>0$. The cable motion thus contributes only positively to (15). However, $u_{A x}$ can either add or subtract from the speed in the $x$ direction in (12), depending on the relative magnitudes of $u_{b}$ and $u_{A x}$ and the sign of $u_{A x}$.

It is important to distinguish between two limiting cases as follows:

1) $u_{b}=0$. In this case, $\left\langle u_{s}\right\rangle^{2}=\left\langle u_{s}^{2}\right\rangle$, since $u_{A x}, u_{A y}$, and $u_{A z}$ all have zero mean values. Alternatively, $\left\langle u_{s}^{\prime 2}\right\rangle=0 .^{2}$

\footnotetext{
${ }^{2}$ Note that in the case $u_{b}=0$, the sensing volume is not flushed by an advecting current, presumably leaving artificially induced wake turbulence. Low current speeds $\left(<0.05 \mathrm{~m} \mathrm{~s}^{-1}\right)$ are routinely flagged and not used for further analysis of turbulence variables.
}

2) $\left.u_{b}^{2}\right\rangle\left\langle u_{A x}^{2}\right\rangle$. In this case all cable motion in the $x$ direction will either increase or decrease the $x$ component of $u_{s}$, but it never fully opposes $u_{b}$ [always $\left(u_{b}+u_{A x}\right)>0$ ], which is why the contribution of $u_{A x}$ averages out, suggesting $\left\langle u_{s}^{\prime 2}\right\rangle \approx\left\langle u_{A x}^{2}\right\rangle$.

We model the fluctuation term in (14) with a weighting function,

$$
\left\langle u_{s}^{\prime 2}\right\rangle=W_{\text {cor }}\left\langle u_{A x}^{2}\right\rangle
$$

where $W_{\text {cor }}$ depends on the relative magnitude of $u_{b}^{2}$ and $\left\langle u_{A x}^{2}\right\rangle$ according to

$W_{\text {cor }}=\left\{\begin{array}{cl}{\left[1-\left(\frac{\left|\left\langle u_{A A}^{2}\right\rangle-u_{b}^{2}\right|}{\left\langle u_{s}^{2}\right\rangle}\right)^{\frac{1}{2}}\right]} & \text { if } u_{b}^{2}<\left\langle u_{A x}^{2}\right\rangle . \\ 1 & \text { otherwise }\end{array}\right.$

Three estimates of $u_{s}$ based on reduced data are compared to the fully resolved $(50-\mathrm{Hz}$ data) estimate $\left\langle u_{s}\right\rangle$ in Fig. 6. These estimates are defined as

$$
\begin{aligned}
& u_{s 1}=\left\langle u_{s}^{2}\right\rangle^{\frac{1}{2}}, \\
& u_{s 2}=\left(\left\langle u_{s}^{2}\right\rangle-\left\langle u_{A x}^{2}\right\rangle\right)^{\frac{1}{2}}, \\
& u_{s 3}=\left(\left\langle u_{s}^{2}\right\rangle-W_{c o r}\left\langle u_{A x}^{2}\right\rangle\right)^{\frac{1}{2}} .
\end{aligned}
$$

We find that $u_{s 1}$, which ignores $\left\langle u_{s}^{\prime 2}\right\rangle$, generally yields an overestimate of about $8 \%$ on average (blue line, Fig. $6 \mathrm{~b}$ ). On the other hand $u_{s 2}$, which always subtracts the entire $\chi$ pod motion contribution in the $x$ direction, results in an underestimate of roughly $5 \%$ on average (red line, Fig. 6b). Our weighted approach, $u_{s 3}$, provides significantly better agreement with the true averaged value, within less than $0.5 \%$ on average (purple line, Fig. 6b), except during the period of anomalously high waves and low currents (16-19 June). This demonstrates that it is sufficient to save only the variance of the three body speed components $\left(\left\langle u_{A x}^{2}\right\rangle,\left\langle u_{A y}^{2}\right\rangle\right.$, and $\left.\left\langle u_{A z}^{2}\right\rangle\right)$ inferred from the three orthogonal acceleration sensors, respectively. These can then be combined with any available mean background velocity $u_{b}$ (in our case, we have used the pitot-static tube, but we could have used the ADCP velocity) to recover the average speed past the sensor $\left\langle u_{s}\right\rangle$.

\section{d. Calibration of body speeds}

We showed in the previous paragraph that the variance of the three body speed components is enough to reconstruct $\left\langle u_{s}\right\rangle$. The remaining problem is to define 


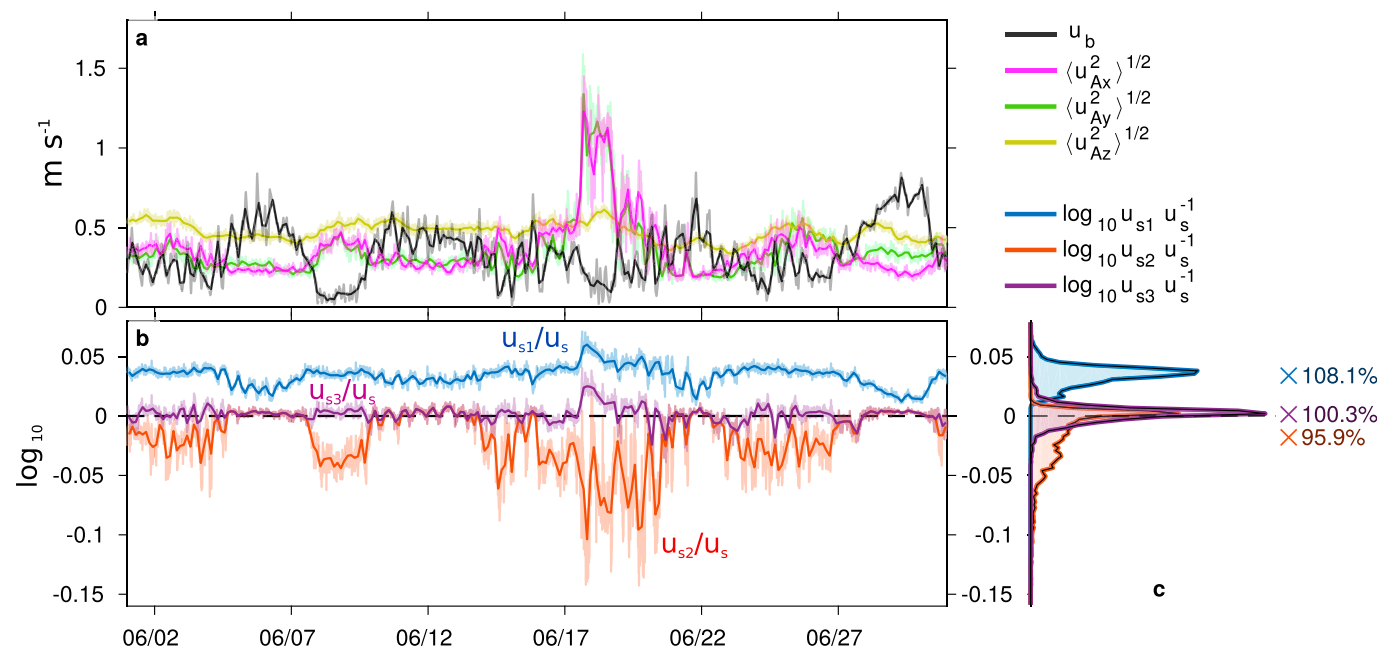

FIG. 6. Comparison of different speeds past the sensor estimates. (a) Time series of the mean current speed (black) and the three components of the $\chi$ pod body speed ( $x$ in purple, $y$ in green, and $z$ in yellow). (b) Time series and (c) distribution of the ratio of the different flow speed past the sensor estimates to the reference (derived from the full 50-Hz data) as well as the mean value (cross). Different estimates correspond to (18), (19), and (20).

these variances from raw voltages that can be computed in preprocessing.

In preprocessing the accelerometer voltage data are integrated,

$$
V_{U z}=\int V_{A z} d t+\text { constant of integration, }
$$

where $V_{U z}$ denotes an uncalibrated signal corresponding to the vertical motion of the $\chi$ pod and the constant of integration is 0 , since the $\chi$ pod goes nowhere. The variance $\left\langle V_{U z}^{2}\right\rangle$ is calculated and stored for transmission.

In postprocessing we make use of the linear calibration relation for the acceleration sensors to calculate the variance of the vertical motion of the $\chi$ pod,

$$
\left\langle u_{A z}^{2}\right\rangle=C_{A z}^{2}\left\langle V_{U z}^{2}\right\rangle,
$$

where $C_{A z}$ is the calibration coefficient for the vertical acceleration component. Analogous relationships are found for the other two motion components, such that we can evaluate $u_{s}$ as in section $5 \mathrm{c}$.

\section{Calibration of temperature and its gradients}

\section{a. Mean temperature}

We typically calibrate temperature as a quadratic function of measured voltage,

$$
T=V_{T}^{2} C_{2 T}+V_{T} C_{1 T}+C_{0 T},
$$

where $T$ is temperature, $V_{T}$ is sensor voltage, and $C_{0-2 T}$ is the corresponding calibration coefficients.

As in (13), we can decompose any time series into an average value $\langle\cdot\rangle$ and the deviation $(\cdot)^{\prime}$,

$$
V_{T}=\left\langle V_{T}\right\rangle+V_{T}^{\prime},
$$

which yields

$$
\left\langle V_{T}^{2}\right\rangle=\left\langle V_{T}\right\rangle^{2}+\left\langle\left(V_{T}^{\prime}\right)^{2}\right\rangle .
$$

Replacing $V_{T}$ in (23) and averaging,

$$
\langle T\rangle=\left[\left\langle V_{T}\right\rangle^{2}+\left\langle\left(V_{T}^{\prime}\right)^{2}\right\rangle\right] C_{2 T}+\langle V\rangle_{T} C_{1 T}+C_{0 T}
$$

TABLE 2. Preprocessing quantities.

\begin{tabular}{lcc}
\hline \multicolumn{1}{c}{ Mean quantities } & Variable & Samples $/ \mathscr{T}$ \\
\hline Time & $\langle t\rangle$ & 1 \\
Mean temperature & $\left\langle V_{T_{1}}\right\rangle,\left\langle V_{T_{2}}\right\rangle$ & 2 \\
Temperature variance & $\left\langle V_{T_{1}}^{2}\right\rangle,\left\langle V_{T_{2}}^{2}\right\rangle$ & 2 \\
Temperature gradient & $\frac{d V_{T_{1}}}{d V_{z}}, \frac{d V_{T_{2}}}{d V_{z}}$ & 2 \\
Acceleration variance & $\left\langle V_{A_{x}}^{2}\right\rangle,\left\langle V_{A_{y}}^{2}\right\rangle,\left\langle V_{A_{z}}^{2}\right\rangle$ & 3 \\
Mean pressure & $\left\langle V_{P}\right\rangle$ & 1 \\
Mean pitot voltage & $\left\langle V_{s}\right\rangle$ & 1 \\
Mean compass & $\left\langle V_{c m p}\right\rangle$ & 1 \\
Spectra reductions & $\Phi_{V_{T 1}}^{\mathrm{BL}}, \Phi_{V_{T_{2}}}^{\mathrm{BL}}$ & $2\left(\Delta f_{\mathrm{IC}} \mathscr{T} / 2\right)[28]^{\mathrm{a}}$ \\
Band limited & $C_{\mathrm{SSF} 1}, C_{\mathrm{SSF} 2}$ & 2 \\
Fitted constant &
\end{tabular}

${ }^{\text {a }}$ For a sampling interval of $\mathscr{T}=600 \mathrm{~s}$ the BL method requires 14 spectral estimates per interval for each of the two temperature sensors, which accounts to a total of 28 . 
in terms of the average voltage $\left\langle V_{T}\right\rangle$ and the voltage variance $\left\langle\left(V_{T}^{\prime}\right)^{2}\right\rangle$. Hence, the quadratic calibration relation requires keeping the variance of the raw signal in addition to the mean to reconstruct $\langle T\rangle$ in postprocessing.

\section{b. Temperature derivative}

After replacing (24) in (23) and subtracting (26), we obtain an equation for the temperature fluctuations,

$$
T^{\prime}=2\left\langle V_{T}\right\rangle C_{2 T} V_{T}^{\prime}+C_{1 T} V_{T}^{\prime}=C_{T p} V_{T}^{\prime},
$$

with the calibration coefficient for the temperature fluctuations

$$
C_{T p}=2\langle V\rangle_{T} C_{2 T}+C_{1 T}
$$

Squaring and averaging of (27) leads to a calibration equation for the temperature variance,

$$
\left\langle\left(T^{\prime}\right)^{2}\right\rangle=\left\langle\left(V_{T}^{\prime}\right)^{2}\right\rangle C_{T p}^{2} .
$$

It also follows that

$$
\frac{d T}{d t}=C_{T p} \frac{d V_{T}}{d t}
$$

And, consequently,

$$
\Phi_{T t}=C_{T p}^{2} \Phi_{V_{T t}}
$$

where $\Phi_{T t}$ and $\Phi_{V_{T t}}$ denote the power spectral density of the time derivative of temperature and of the uncalibrated temperature sensor voltage, respectively.

As a test of the calibration procedure, Fig. 7a shows a comparison between the spectra of the calibrated temperature time series and the postcalibrated spectra according to (31) for the entire month. The error generated by the postcalibration is, on average, on the order of $0.01 \%$ and therefore negligible.

\section{c. Temperature stratification}

As pointed out in section 2, we also need to recover the mean vertical temperature gradient, because it yields the last two primary ingredients for our $\chi$ pod calculations. For $\chi$ pods deployed on surface-pumped moorings, the vertical temperature gradient can be calculated internally in the $\chi$ pod by fitting the vertical displacement against the measured temperature (Perlin and Moum 2012),

$$
T(z)=T_{z} z_{\chi}+\langle T\rangle
$$

where $z_{\chi}$ is the vertical displacement of the $\chi$ pod. The displacement $z_{\chi}$ can be determined either by making
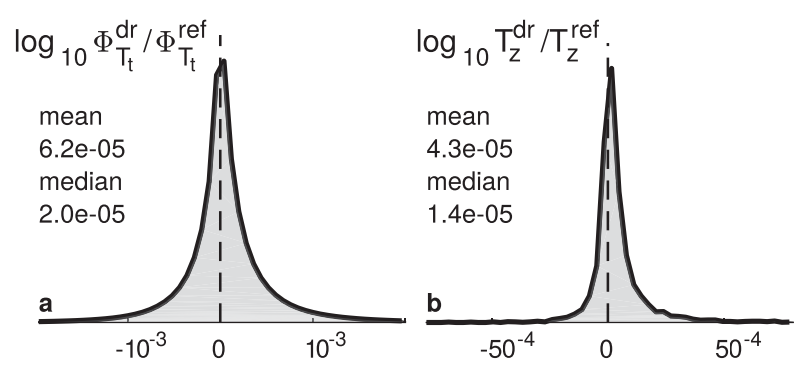

FIG. 7. Temperature spectra and gradient calibrations. (a) Ratio of spectral estimates obtained from (31) $\Phi_{T_{t}}^{\mathrm{dr}}$ compared to spectral estimates based on precalibrated temperature data $\Phi_{T_{t}}^{\text {ref }}$. (b) Ratio of the vertical temperature gradient calculated with (33) $T_{z}^{\mathrm{dr}}$ and based on precalibrated data $T_{z}^{\text {ref }}$. Both ratios are calculated using the data from the entire month.

use of the internal pressure sensor or by double integration in time of the vertical accelerometer (Perlin and Moum 2012).

To calculate $T_{z}$ in the context of data reduction, we perform double integration in time of the raw signal of the vertical acceleration component [similar to (21)], to obtain the vertical displacement in terms of $V_{z}$. Then we fit the raw voltage of temperature against $V_{z}$ on board the $\chi$ pod and retain the slope, which afterward can be calibrated according to

$$
T_{z}=\frac{C_{T p}}{C_{A z}} \frac{d V_{T}}{d V_{z}},
$$

where we made use of (28). Figure $7 \mathrm{~b}$ illustrates that the error in $T_{z}$ due to the postcalibration is very small.

\section{Compression of spectral information}

We have seen in section $6 b$ that it is possible to calibrate a raw voltage spectrum to reliably recover a full temperature spectrum. However, the full 10-min spectrum contains more information than needed to obtain a robust estimate of $\chi$. Here we investigate two progressively sparse procedures to approximate the temperature gradient spectra needed to estimate $\chi$.

1) In the first procedure, only spectral coefficients from the IC range are retained; that is, a band-limited (BL) spectral representation is retained.

2) In the second procedure, the amplitude of the $f^{1 / 3}$ range is determined by a spectral slope fit (SSF), thus reducing the spectral information to a single value.

\section{a. BL spectra}

We consider only spectral coefficients within the IC band but at frequencies lower than surface waves $\left(\Delta f_{\mathrm{IC}}=1 / 200 \ldots 1 / 20 \mathrm{~Hz}\right)$. This corresponds to 14 coefficients 
TABLE 3. Data rate (bytes per hour) required by the VC, BL, and SSF methods as a function of averaging interval $\mathscr{T}$.

\begin{tabular}{cccr}
\hline \hline Averaging interval (s) & VC estimate & BL & SSF \\
\hline $\mathscr{T}=600$ & 4348800 & 492 & 180 \\
$\mathscr{T}=1200$ & 4348800 & 414 & 90 \\
$\mathscr{T}=2400$ & 4348800 & 375 & 45 \\
$\mathscr{T}=3600$ & 4348800 & 362 & 30 \\
\hline
\end{tabular}

per 10-min spectrum, which accounts to 28 for both temperature sensors combined (see Table 2).

In practice, this means preprocessing of 10-min spectra of the raw signal for each of the two temperature sensors. Only the spectral coefficients that fall into the IC-fitting band $\Delta \mathrm{f}_{\mathrm{IC}}$ are retained for postprocessing.

The narrowband raw spectra are then postcalibrated using (31) to obtain $\Phi_{T_{t}}^{\mathrm{BL}}$. By combining $\Phi_{T_{t}}^{\mathrm{BL}}$ and $u_{s}$, we obtain $\Phi_{\mathrm{T}_{\mathrm{x}}}^{\mathrm{BL}}$ (blue line, Fig. 8), which is then used to estimate $\chi_{\mathrm{BL}}$.

\section{b. $S S F$}

The amplitude of the best fit of the slope $f^{1 / 3}$ to the IC range of the preprocessed spectrum is determined and then used to compute $\chi_{\mathrm{SSF}}$. This procedure packages the entire temperature spectrum information into a single number (see Table 2). Additionally, it is possible to return the fitted slope of the observed data spectrum, to allow for a measure of quality control by determining its deviation from $1 / 3$.

As in the IC-band limitation scheme, preprocessing requires computation of the power spectral density of the raw voltage of the temperature derivative in frequency space, $\Phi_{V_{T t}}(f)$, over a 10 -min window.

In the second step of preprocessing, it is assumed that $\Phi_{V_{T t}}(f)$ can be modeled with a $1 / 3$ power law in the IC band (see Batchelor 1959; Kraichnan 1968),

$$
\Phi_{V_{T_{t}}}(f)=C_{\mathrm{SSF}} f^{1 / 3},
$$

where we introduce the constant $C_{\mathrm{SSF}}$,

$$
C_{\mathrm{SSF}}=\left\langle\frac{\Phi_{V_{T_{t}}(f)}}{f^{1 / 3}}\right\rangle_{\left[\frac{1}{200}<f<\frac{1}{20} \mathrm{~Hz}\right]},
$$

with $\langle\cdot\rangle_{f}$ denoting a frequency-band average over the IC-fitting band $\left(\Delta f_{\mathrm{IC}}\right)$. Computation of $\Phi_{V_{T_{t}}}$ and $C_{\mathrm{SSF}}$ are done in preprocessing, such that the spectrum is reduced to a single number $C_{\mathrm{SSF}}$.

In postprocessing the spectral parameter $C_{\mathrm{SSF}}$, in combination with $u_{s}, T_{z}, N^{2}$, and sensor-specific calibration coefficients, is translated into the calibrated turbulence quantities. We first develop the relation between these quantities and $\chi_{\mathrm{SSF}}$ and $\varepsilon_{\mathrm{SSF}}$.

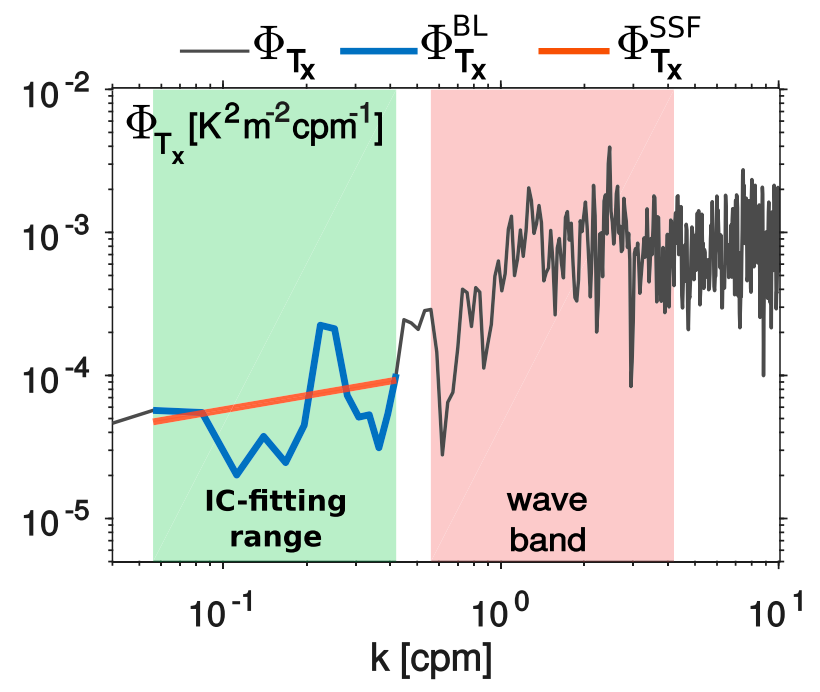

FIG. 8. Randomly chosen temperature gradient spectrum in wavenumber space. Full 10-min spectrum (black), partly in the IC-fitting range (blue), and the $1 / 3$ slope fit (red). Background shading indicates the IC-fitting range (corresponding to 20-200s, green) and the surface wave band (red).

The temperature derivative spectrum in wavenumber space is related to that in frequency space by

$$
\begin{aligned}
\Phi_{T t}(k) & =\Phi_{T t}(f) \frac{d f}{d k} \\
& =\left(\frac{\left\langle u_{s}\right\rangle}{2 \pi}\right)^{4 / 3} C_{\mathrm{SSF}} C_{T p}^{2} k^{1 / 3},
\end{aligned}
$$

where in the last step we made use of (31), (34), and (7) Via (6) and (36),

$$
\begin{aligned}
\Phi_{T_{x}}(k) & =\left\langle u_{s}\right\rangle^{-2} \Phi_{T t}(k) \\
& =\left\langle u_{s}\right\rangle^{-2 / 3}(2 \pi)^{-4 / 3} C_{\mathrm{SSF}} C_{T p}^{2} k^{1 / 3} .
\end{aligned}
$$

With (11) it is possible to reformulate the first term on the rhs of (8) (the IC range) as a function of $\chi$ :

$$
\Phi_{T_{x}}(k)=C_{\tau} \chi^{2 / 3}\left(\frac{N^{2}}{2 \Gamma T_{z}^{2}}\right)^{1 / 3} k^{1 / 3}
$$

or $\varepsilon$ only:

$$
\Phi_{T_{x}}(k)=C_{\tau} \varepsilon^{2 / 3}\left(\frac{N^{2}}{2 \Gamma T_{z}^{2}}\right)^{-1} k^{1 / 3} .
$$

Combining (37) and (38) yields

$$
\chi_{\mathrm{SSF}}=\frac{C_{T p}^{3}}{\left\langle u_{s}\right\rangle(2 \pi)^{2}}\left(\frac{C_{\mathrm{SSF}}}{C_{\tau}}\right)^{3 / 2}\left(\frac{N^{2}}{2 \Gamma T_{z}^{2}}\right)^{-1 / 2},
$$


and analogously with (40),

$$
\varepsilon_{\mathrm{SSF}}=\frac{C_{T p}^{3}}{\left\langle u_{s}\right\rangle(2 \pi)^{2}}\left(\frac{C_{\mathrm{SSF}}}{C_{\tau}}\right)^{3 / 2}\left(\frac{N^{2}}{2 \Gamma T_{z}^{2}}\right)^{3 / 2},
$$

which are the key equations for putting our ingredients listed in section 2 together.

In summary the postprocessing steps are as follows:

1) reconstruct $u_{s}$ via (20),

2) use (33) to obtain $T_{z}$,

3) infer $N^{2}$ from $T_{z}$, the temperature gradient alone, ${ }^{3}$

4) and, finally, combine $u_{s}, T_{z}$, and $N^{2}$ with $C_{\text {SSF }}$ via (40) and (41) to obtain $\chi$ and $\varepsilon$, respectively. ${ }^{4}$

\section{Evaluation}

In the following, we evaluate the performance of the reduction schemes introduced in section 7 . Each is combined with $u_{s 3}$ from (20) and $T_{z}$ from (33), and $N^{2}$ is estimated as $-g \alpha T_{z}$. Thus, the resulting $\chi_{\mathrm{BL}}$ and $\chi_{\mathrm{SSF}}$ are purely based on our data reduction products. To test their performance we use $\chi_{\mathrm{IC}}$ as a reference, which we obtain utilizing the full $100-\mathrm{Hz} \chi$ pod information for $\Phi_{T_{t}}, u_{s}, T_{z}$, and $N^{2}$, respectively (Fig. 9). This comparison therefore illustrates how much the estimates deviated purely as a result of data reduction.

A direct comparison of estimates as time series is difficult (see Fig. 9a), and the differences can only be seen in ratios of the two estimates to $\chi_{\mathrm{IC}}$ (Fig. 9b). Even in Fig. 9b there are only three time spans where the two estimates diverge significantly from $\chi_{\mathrm{IC}}$. These are typically at low values of $\chi_{\mathrm{IC}}$, where $\chi_{\mathrm{SSF}}$ slightly overestimates $\chi_{\mathrm{IC}}$.

In general we find that both estimates correlate highly $(>99.7 \%)$ with $\chi_{\text {IC }}$ (Figs. 9c,d) and that their averages and median deviate only marginally (Fig. 9e). When comparing the two estimates in detail to $\chi_{\mathrm{IC}}$ (Figs. 9f,g), some differences can be identified. The overwhelming part of $\chi_{\mathrm{BL}}$ does not deviate from $\chi_{\mathrm{IC}}$ by more than $10 \%$ with no significant bias. Given the fact that the postcalibration of the spectrum and vertical temperature gradient introduce almost no error (sections $6 \mathrm{~b}, \mathrm{c})$, the deviation of $\chi_{\mathrm{IC}}$ and $\chi_{\mathrm{BL}}$ must be primarily due to errors in our estimate of $u_{s}$ (section $5 \mathrm{c}$ ). Also, $\chi_{\mathrm{SSF}}$ compares well with $\chi_{\mathrm{IC}}$, although $\chi_{\mathrm{SSF}}$ tends to

\footnotetext{
${ }^{3}$ This assumes that salinity stratification can be neglected. If this is not the case, then additional salinity information from other instruments must be included.

${ }^{4}$ Note that the high-frequency temperature measurements are reduced to a single value $C_{\mathrm{SSF}}$ representing a 10 -min time series sampled at $100 \mathrm{~Hz}$.
}

overestimate $\chi_{\mathrm{IC}}$ for small $\chi_{\mathrm{IC}}$ (Fig. $9 \mathrm{~g}$ ). Similar to $\chi_{\mathrm{BL}}$, the average bias of $\chi_{\mathrm{SSF}}$ is very small (Fig. 9e).

Figure 10 shows the ensemble of 10-min spectral estimates from the entire month, where we averaged spectra with ranges of comparable $\chi$ values. For spectra represented by $\chi>10^{-7} \mathrm{~K}^{2} \mathrm{~s}^{-1}$, the IC range is characterized by a clear $1 / 3$ slope (red line), which also means that both of our reduction schemes agree. Revisiting Fig. 2, when $\chi>10^{-7} \mathrm{~K}^{2} \mathrm{~s}^{-1}$, there is also generally good agreement between the IC and VC estimates. For these times we can therefore conclude that the Batchelor theory consistently describes the low- and high-frequency parts of the spectrum.

At lower $\chi$ values, there is significant disagreement between the slopes of measured spectra and $f^{1 / 3}$ (cf. lower two blue and red lines in Fig. 10). The average slope in the IC-fitting range tends to smaller and even negative values with decreasing $\chi$. This trend is seen in Fig. 11b, showing the distribution of all $\chi$ values with respect to a best-fit slope in the IC-fitting range. While most slope values cluster around the theoretical values of $1 / 3$, we find it to systematically decrease with diminishing $\chi$. Figure 11a furthermore shows that at times where the observed slope in the IC-fitting range deviates most from $1 / 3$, the IC and VC estimates also disagree the most. Therefore, the best-fit slope in the IC-fitting range seems to provide a useful quality control for the turbulence estimates obtained by the data reduction scheme. It can be computed in preprocessing and then transmitted together with $C_{\mathrm{SSF}}$, as a quality control parameter.

We summarize the data volumes required by each reduction scheme in Table 3, which shows estimates of the required data volume per hour where each sample is represented by a 2-byte word. Although many more data are stored on a $\chi$ pod, the actual amount of data required to perform the standard procedure to estimate $\chi_{\mathrm{VC}}$ accounts to about 4 megabytes per hour. Even with the less efficient reduction scheme BL, this number is reduced by four orders of magnitude to about 500 bytes per hour. The onboard scaling procedure (SSF) reduces the data volume by another factor of 3 and is thus the most efficient reduction scheme, requiring about 180 bytes per hour on 10-min averages, or 24000 times less data than the standard procedure. Given the fact that both reduction schemes perform almost equally well, we find no obvious reason not to use the SSF procedure.

\section{Applicability of IC scaling in an anisotropic regime}

An assessment of the frequency range over which we fit $\Phi_{\mathrm{Tx}}$ (Fig. 12b) shows that it is always higher than 

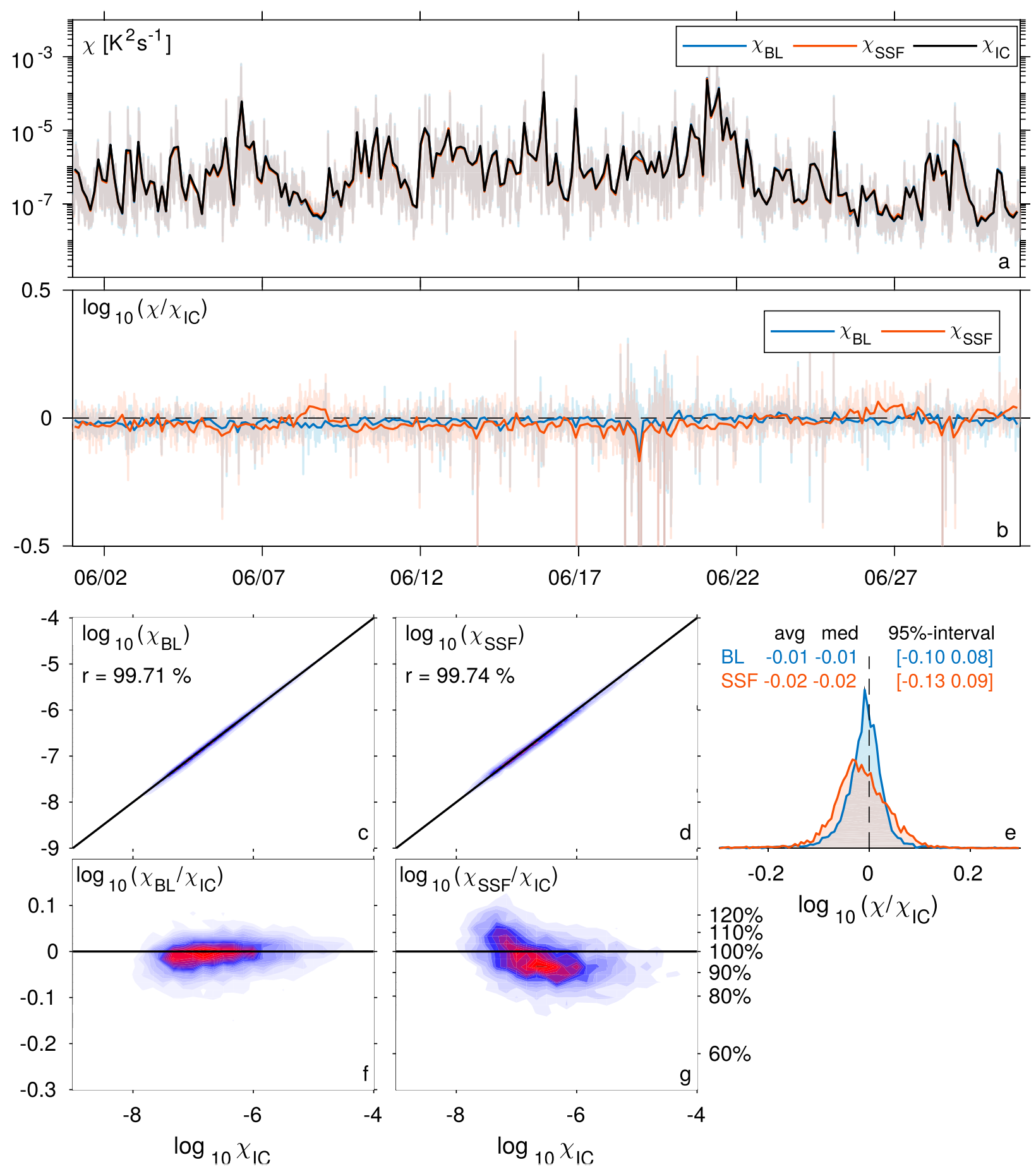

FIG. 9. (a) Time series of $\chi$ based on the two different data reduction methods, with the band limitation (blue) and the fitting procedure (red), and as a reference the IC estimates based on the fully calibrated data from Fig. 2. (b) Time series of the ratio of the different estimates with respect to the reference. Shown in (a) and (b) are 10-min values (shading) and 3-h moving averages (shading). (c), (d) Correlation of the three reduction methods with the reference IC estimate, and the (e) corresponding 1D and (f), (g) 2D distributions of the ratios, calculated with the 10 -min estimates. 


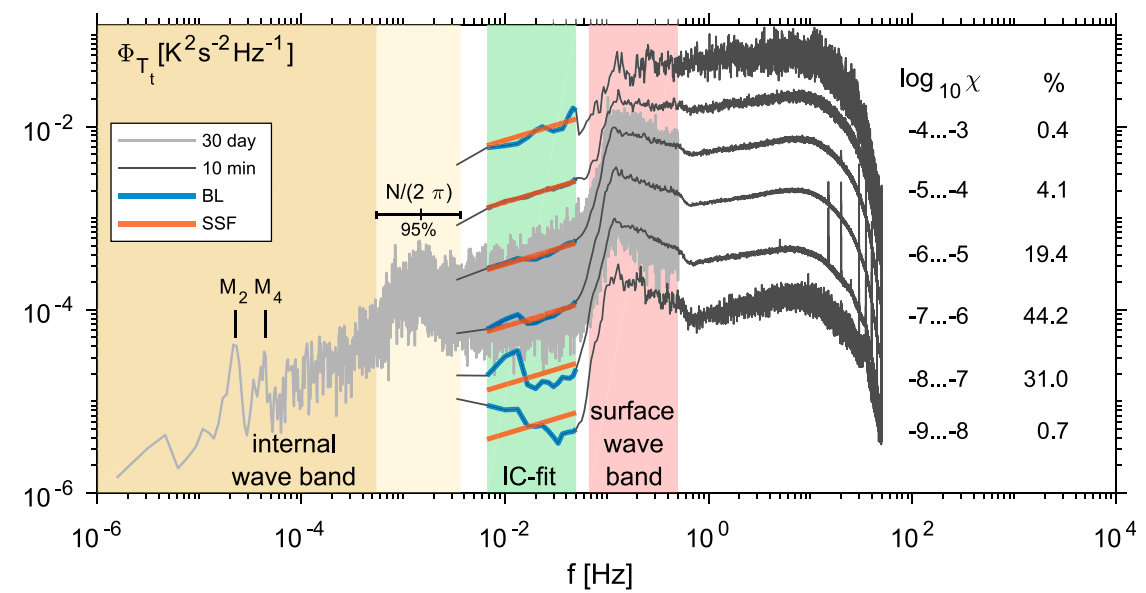

FIG. 10. Temperature derivative spectra. The 10-min spectra ensemble (black lines) averaged in $\chi$ ranges indicated to the right. Relative number of 10 -min samples in each range is also indicated. Two data reduction schemes are shown: BL (blue line) and SSF (red lines). Spectrum computed from the entire one-month record (light gray line). Color shading in the background indicates the following: internal wave (yellow) and surface wave frequency bands (red), the $1 / 200-1 / 20-\mathrm{Hz}$ band used for inertial-convective range fits (green), and the $95 \%$ range of $N /(2 \pi)$, the theoretical lower-frequency limit for internal waves (light yellow).

the local value of $N / 2 \pi$. This means that we do not risk fitting the spectra over the internal wave band of frequencies. Another relevant metric is the frequency associated with the advection of the largest turbulent eddies past the sensors. This is related to the Ozmidov scale, $L_{\mathrm{Oz}}=\left(\varepsilon / N^{3}\right)^{1 / 2}$, where the equivalent frequency is $f_{\mathrm{Oz}}=u_{b} /\left(2 \pi L_{\mathrm{Oz}}\right)$. Term $L_{\mathrm{Oz}}$ is usually interpreted as a vertical limit for the growth of turbulent eddies inferred by vertical stratification (Gargett et al. 1984). It can be understood as a scale above which turbulence fluctuations are anisotropic. Despite the fact that the Batchelor theory assumes isotropy, Brethouwer et al. (2007) have demonstrated that inertial subrange scalings can be found in horizontal velocity and tracer spectra that extend to length scales that are orders of magnitude larger than $L_{\mathrm{Oz}}$. Hence,
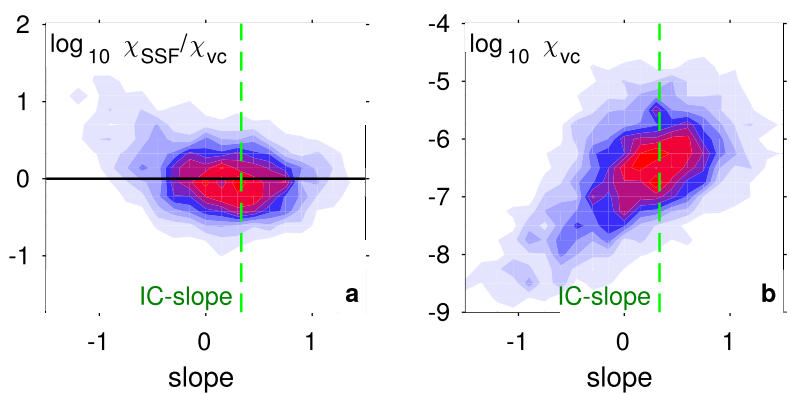

FIG. 11. 2D histograms. (a) Ratio between the $\chi_{\mathrm{SSF}}$ and the bestfit slope in log space for the IC range of the measured spectrum. Note that the $\chi_{\mathrm{SSF}}$ procedure implies a 1/3 slope (dashed green line). (b) Comparison of the best-fit slope in the IC range and the $\chi$ estimate from the $\mathrm{VC}$ range scaling. even though we may be fitting spectra over a range of frequencies that does not represent isotropic turbulence, the results (when compared to VC estimates, Fig. 12a) are in reasonably agreement.

\section{Conclusions}

We have developed pre- and postprocessing procedures to reduce the data required to reliably compute quantitative turbulence variables from $x$ pod measurements. In

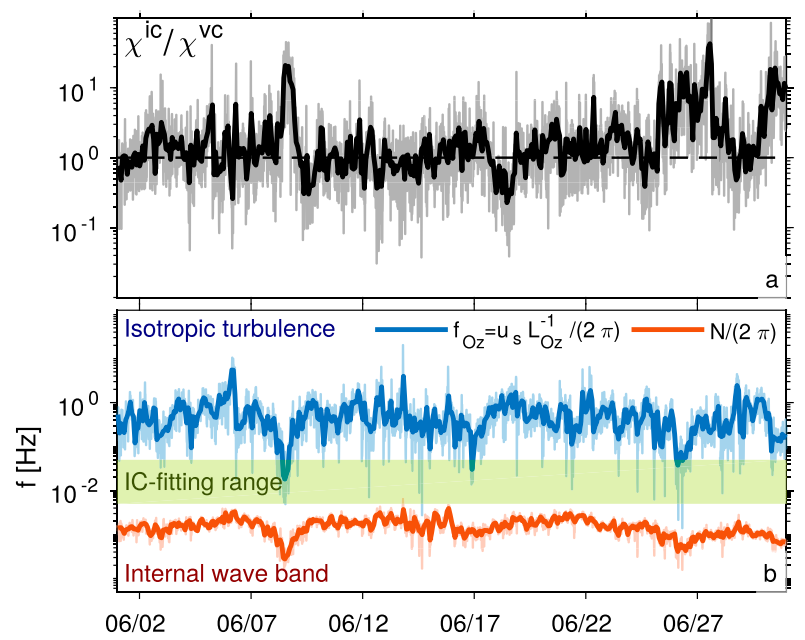

FIG. 12. (a) Ratio between the IC and VC $\chi$ estimates. (b) Comparison of the frequency range of our inertial-convective fits (green) to buoyancy frequency (orange) and to the equivalent advected frequency of $L_{\mathrm{Oz}}$ (blue). 
comparison to reference estimates of $\chi$ that employ the complete dataset over 8640 individual 10-min estimates, our most efficient (the method that maximally reduces the data) computation is biased low, on average by $5 \%$ with a $95 \%$ range of values $0.74-1.25$ times the reference (Fig. 9e).

These procedures require a volume of data that is orders of magnitude smaller than the complete dataset we have been typically using for processing. The necessary data volume is small enough to be transferred via satellite, which potentially allows for real-time data monitoring and additionally provides valuable data backup in case instruments are lost or are heavily damaged during deployment. It also potentially leads to deployments that do not include the option of recovery.

The method developed in this study demonstrates how the $\chi$ pod can be used as a fully self-contained device that is independent of additional instrumentation, such as ADCPs. It provides independent measures of temperature, pressure, acceleration, vertical stratification (in case of negligible salinity gradient), ${ }^{5}$ velocity, and $\chi$, plus derived quantities $\varepsilon, K_{T}$, buoyancy flux, etc. These quantities are reconstructed at 10 -min averages from a preprocessed data volume of 180 bytes per hour.

Acknowledgments. We are grateful to two anonymous reviewers, who helped with their comments to improve the manuscript. This work was funded by the National Science Foundation $(1256620,1431518)$ and the Office of Naval Research (N00014-15-1-2466). Pavan Vutukur, Craig Van Appeldorn, and Mike Neeley-Brown were responsible for the successful operation of $\chi$ pods. Bernard Bourles and colleagues at the Laboratoire d'Études en Geophysique et Océanographie Spatiales of the Institut de Recherche pour le Développement (IRD), France, and Peter Brandt and colleagues at the GEOMAR Helmholtz Centre for Ocean Research Kiel, Germany, have been most gracious in accommodating $\chi$ pods on their moorings. We are also grateful to NOAA's PMEL personnel for helping with many aspects of these deployments.

\section{REFERENCES}

Batchelor, G., 1959: Small-scale variation of convected quantities like temperature in turbulent fluid part 1. General discussion and the case of small conductivity. J. Fluid Mech., 5, 113-133, https://doi.org/10.1017/S002211205900009X.

Bourles, B., and Coauthors, 2008: The PIRATA program: History, accomplishments, and future directions. Bull. Amer. Meteor. Soc., 89, 1111-1125, https://doi.org/10.1175/2008BAMS2462.1.

\footnotetext{
${ }^{5}$ When and where salinity contributes significantly to $N_{2}$, an independent measurement is required.
}

Brethouwer, G., P. Billant, E. Lindborg, and J.-M. Chomaz, 2007: Scaling analysis and simulation of strongly stratified turbulent flows. J. Fluid Mech., 585, 343-368, https://doi.org/10.1017/ S0022112007006854.

Gargett, A. E., T. Osborn, and P. Nasmyth, 1984: Local isotropy and the decay of turbulence in a stratified fluid. J. Fluid Mech., 144, 231-280, https://doi.org/10.1017/S0022112084001592.

Klymak, J. M., and J. N. Moum, 2007: Oceanic isopycnal slope spectra. Part II: Turbulence. J. Phys. Oceanogr., 37, 1232-1245, https://doi.org/10.1175/JPO3074.1.

Kraichnan, R. H., 1968: Small-scale structure of a scalar field convected by turbulence. Phys. Fluids, 11, 945-953, https:// doi.org/10.1063/1.1692063.

Moum, J. N., 2015: Ocean speed and turbulence measurements using pitot-static tubes on moorings. J. Atmos. Oceanic Technol., 32, 1400-1413, https://doi.org/10.1175/ JTECH-D-14-00158.1.

— , and J. D. Nash, 2009: Mixing measurements on an equatorial ocean mooring. J. Atmos. Oceanic Technol., 26, 317-336, https://doi.org/10.1175/2008JTECHO617.1.

,-- , and W. D. Smyth, 2011: Narrowband oscillations in the upper equatorial ocean. Part I: Interpretation as shear instabilities. J. Phys. Oceanogr., 41, 397-411, https://doi.org/ 10.1175/2010JPO4450.1.

_ A. Aerlin, J. D. Nash, and M. J. McPhaden, 2013: Seasonal sea surface cooling in the equatorial Pacific cold tongue controlled by ocean mixing. Nature, 500, 64-67, https://doi.org/10.1038/ nature12363.

—, K. Pujiana, R.-C. Lien, and W. Smyth, 2016: Ocean feedback to pulses of the Madden-Julian Oscillation in the equatorial Indian Ocean. Nat. Commun., 7, 13203, https://doi.org/ 10.1038/ncomms13203.

Osborn, T. R., 1980: Estimates of the local rate of vertical diffusion from dissipation measurements. J. Phys. Oceanogr., 10, 83-89, https:// doi.org/10.1175/1520-0485(1980)010<0083:EOTLRO > 2.0.CO;2.

_ and C. S. Cox, 1972: Oceanic fine structure. Geophys. Fluid Dyn., 3, 321-345, https://doi.org/10.1080/03091927208236085.

Perlin, A., and J. Moum, 2012: Comparison of thermal variance dissipation rates from moored and profiling instruments at the equator. J. Atmos. Oceanic Technol., 29, 1347-1362, https:// doi.org/10.1175/JTECH-D-12-00019.1.

Pope, S. B., 2000: Turbulent Flows. Cambridge University Press, $771 \mathrm{pp}$.

Smyth, W., and J. Moum, 2013: Marginal instability and deep cycle mixing in the eastern equatorial Pacific Ocean. Geophys. Res. Lett., 40, 6181-6185, https://doi.org/10.1002/2013GL058403.

$\ldots, \ldots$, and J. Nash, 2011: Narrowband high-frequency oscillations at the equator. Part I: Interpretation as shear instabilities. J. Phys. Oceanogr., 41, 412-428, https://doi.org/10.1175/2010JPO4451.1.

Sreenivasan, K. R., 1996: The passive scalar spectrum and the Obukhov-Corrsin constant. Phys. Fluids, 8, 189-196, https:// doi.org/10.1063/1.868826.

Taylor, G. I., 1935: Statistical theory of turbulence. Proc. Roy. Soc. London, 151A, 421-444, https://doi.org/10.1098/rspa.1935.0158.

Warner, S., J. Becherer, K. Pujiana, E. Shroyer, M. Ravichandran, V. Thangaprakash, and J. Moum, 2016: Monsoon mixing cycles in the Bay of Bengal: A year-long subsurface mixing record. Oceanography, 29 (2), 158-169, https://doi.org/10.5670/ oceanog.2016.48.

Zhang, Y., and J. N. Moum, 2010: Inertial-convective subrange estimates of thermal variance dissipation rate from moored temperature measurements. J. Atmos. Oceanic Technol., 27, 1950-1959, https://doi.org/10.1175/2010JTECHO746.1. 